\title{
Account
}

\section{Nonlinear Optical Zeolite Films for Second and Third Harmonic Generation}

\author{
Hyun Sung Kim, Tung Thanh Pham, and Kyung Byung Yoon*
Korea Center for Artificial Photosynthesis, Center for Nano Materials, and Department of Chemistry, Sogang University, Seoul 121-742, Korea. E-mail: yoonkb@sogang.ac.kr
Received March 1, 2011, Accepted April 19, 2011

\begin{abstract}
Methods to prepare novel second-order nonlinear optical (2O-NLO) materials composed of all-silica zeolite (silicalite-1) and a series of 2O-NLO molecules having high second order hyperpolarizability constants ( $\beta$ values) are reviewed. These methods include the development of novel methods to incorporate a series of hemicyanine (HC) molecules into the channels of silicaite-1 films in uniform orientations. The first method is to incorporate $\mathrm{HC}$ molecules tethered with long alkyl chains (octadecyl or longer) into the silicalite-1 channels with the long alkyl chain side first through the hydrophobic-hydrophobic interaction between the long alky chains and the silicalite- 1 channels. The second method is to incorporate the HC molecule tethered with a medium length alkyl chain (nonyl) into the silicalite-1 channels with the medium length alkyl chain side first through hydrophobic-hydrophobic interaction between the medium length alky chain in the photoexcited state and the silicalite- 1 channels. The third method is to incorporate the HC molecule tethered with propionic acid into the silicalite- 1 channels with the propionic acid side last mediated by a tetrabultylammonium cation ionpaired to the propionate unit. A method to prepare a novel third-order nonlinear optical (3O-NLO) material composed of zeolite-Y and $\mathrm{PbS}$ or $\mathrm{PbSe}$ quantum dots is also reviewed. This Account thus describes a promising new direction to which the search for highly sensitive 2O-NLO and 3O-NLO materials has to be conducted and a new direction to which zeolite research and applications have to be expanded.
\end{abstract}

Key Words : Zeolite, Nonlinear optical film, Hemicyanine, PbS, PbSe quantum dots

\section{Introduction}

A large number of organic dipolar nonlinear optical (NLO) dyes with high second-order (2O) hyperpolarizability constants ( $\beta$-values) has been synthesized. The conventional methods to prepare $2 \mathrm{O}-\mathrm{NLO}$ materials based on the organic 2O-NLO dyes can be classified into (1) the crystallization of the 2O-NLO dyes, (2) the preparation of 2O-NLO dyepolymer physical mixtures or preparation of 2O-NLO dyetethering polymers on optically transparent supports followed by electrical poling, ${ }^{1,2,9-13}$ (3) the formation of selfassembled mono- or multilayers of 2O-NLO dyes on optically transparent supports, ${ }^{4-8}$ and (4) the incorporation of $2 \mathrm{O}-\mathrm{NLO}$ dyes into large $(\sim 130 \mu \mathrm{m})$ zeolite powders or the powders of the related nanoporous materials.
Each method has its own merits and demerits. The demerits which have not allowed them to be used for practical applications are as follows. In the case of the crystallization method, the strong tendency of the 2O-NLO dyes to align centrosymmetrically within the crystals is the most significant demerit and because of this most of the obtained crystals do not show the $2 \mathrm{O}-\mathrm{NLO}$ activity. There have been large amounts of efforts to break the centrosymmetry during their crystallization. However, most of such efforts have been shown to be unsuccessful except some limited cases. ${ }^{1-3}$ In the case of the electrically poled 2O-NLO dye-polymer composite materials, the low 2O-NLO activity and the loss of activity with time arising from the thermal instability have been the major demerits. In the case of self-assembled monoor multilayers of 2O-NLO dyes, no practical applicability
Kyung Byung Yoon received his B.S. in 1979 from the Department of Chemistry, Seoul National University. In 1981, he obtained his M.S. from the Department of Chemistry, Korea Advanced Institute of Science and Technology (KAIST), Seoul. From 1981 to 1984 he was employed by Chon Engineering Co. LTD, Seoul, Korea. In 1989, he earned his Ph.D. degree from the Department of Chemistry, University of Houston. He has been an Assistant (1989), Associate (1993) and Full Professor (1998) in Sogang University. He is currently serving as the Dean of the College of Natural Science and the Director of the Korea Center for Artificial Photosynthesis (KCAP) located in Sogang University. He served as the Vice President of the Korean Chemical Soci- ety (KCS) in 2001 and 2005 and the Audit in 2010. He is currently the Chairman of the Inorganic Chemistry Division in KCS and the President of the Korea Photoscience Association. During 2005-2007, he served as the Secretary General of the Federation of Asian Chemical Societies (FACS). He also holds the Councillorships for the International Zeolite Association, the Asian-Oceanian Photochemistry Association, and FACS. He received 'Taikyue Ree Academic Award' in 2007 from KCS, 'Academic Award' in 2008 from National Academy of Science, and 'Korea Science Award' in 2010 from the Republic of Korea. 
arising from mechanical instability have been the problems. In the case of 2O-NLO dye-incorporating zeolite powders, only the 2O-NLO dyes with relatively low $2 \mathrm{O}$ hyperpolarizability constants ( $\beta$ values) ${ }^{14,15}$ have been shown to enter the zeolite micro crystals with uniform orientations, giving rise to low $2 \mathrm{O}-\mathrm{NLO}$ activities. ${ }^{16-24}$

We have developed a novel method which is free from the demerits described above. ${ }^{25-27}$ The key idea of this method is to incorporate 2O-NLO dyes with uniform orientations into zeolite films supported on optically transparent substrates. The produced 2O-NLO dye-incorporating zeolite films have shown much higher thermal and mechanical stability than those of the conventional methods and no loss of activity with time, bearing a great potential to be practically applied in industry. This Account summaries the details of the method developed in our laboratory and the future perspectives.

Semiconductor quantum dots (QDs) dispersed in glass and polymer matrices have shown third-order (3O) NLO properties. ${ }^{28-39}$ However, their sensitivities have not yet reached the degree that is sufficient for practical applications. Zeolites are also excellent hosts for QDs ${ }^{40-51}$ and have many advantages over glass and polymers due to the followings. The zeolite-encapsulated QDs are usually smaller than $1.5 \mathrm{~nm}$, their sizes are highly uniform, and they exist very regularly within the crystalline hosts. We showed that zeoliteencapsulated $\mathrm{PbS}$ QDs show very high 3O-NLO activities. ${ }^{50}$ This Account also summaries the methods to prepare $\mathrm{PbS}$ QD-incorporating zeolite Y (Fig. 1) films supported on glass, the 3O-NLO characteristics of the prepared films, and the future perspectives.

Previous Efforts to Prepare 2O-NLO Zeolite Powders. The tested 2O-NLO dyes were para-nitroaniline, 2-methyl4-nitroaniline, 2-amino-4-nitropyridine, 4-nitro- $N, N$-dimethylaniline, ${ }^{19}$ and (dimethylamino)benzonitrile. ${ }^{20}$ The studied nanoporous materials were $\mathrm{AlPO}_{4}-5$ having one-dimensional straight channels with the diameter of $0.8 \mathrm{~nm}, \mathrm{ZSM}-5^{21}$ and Sb-incorporating silicalite- $1,{ }^{22}$ centrosymmetric (Pnma) zeolites having a three-dimensional channel system consisting of straight $0.54 \times 0.56 \mathrm{~nm}$ channels along the $b$ axis and sinusoidal $0.51 \times 0.54 \mathrm{~nm}$ channels along the a axis, pure (a)

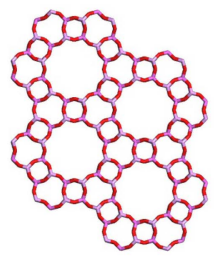

$7.3 \AA$

(d)

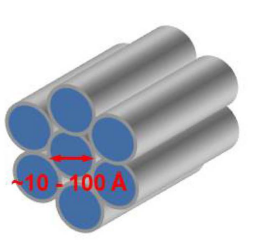

(b)

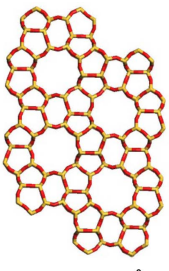

$5.3 \times 5.6 \AA$ (c)

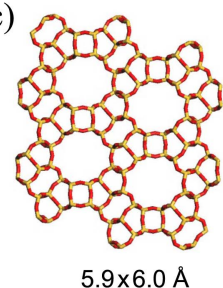

(e)

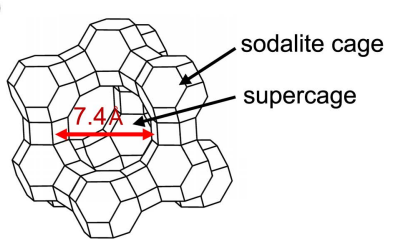

Figure 1. Structures and pore sizes of $\mathrm{AlPO}_{4}-5$ (a), ZSM-5 or silicalite-1 (b), ZSM-12 (c), MCM-41 (d), and zeolite-Y (e).

silica ZSM-12, a centrosymmetric (C2/c) zeolite, having one-dimensional channels with the diameter of $0.56 \times 0.59$ $\mathrm{nm}$, and MCM-41, an amorphous silica having a hexagonal array of channels with the diameter of 2-8 nm (Fig. 1).

The dye-incorporating $\mathrm{AlPO}_{5}-5$ powders generate the second harmonic $(\mathrm{SH})$ with the intensity far exceeding that of quartz powders. ${ }^{16,17}$ The SH-generating (SHG) activity arose as a result of the spontaneous inclusion of paranitroaniline into the $\mathrm{AlPO}_{4}-5$ channels with the nitro group first caused by the intrinsically higher affinity of the $\mathrm{AlPO}_{4}-$ 5 channels to nitro than amino group. ${ }^{18}$ Since the sizes of the crystals far exceeded (such as $130 \mu \mathrm{m}$ ) the wavelength of the incident laser beam $(1.064 \mu \mathrm{m})$, the polarization reversal that occurs at the center of each crystal does not affect the overall SHG activities of the dye-loaded $\mathrm{AlPO}_{4}-5$ crystals. The $\mathrm{ALPO}_{4}-5$ crystals loaded with 4-nitro- $N, N$-dimethylaniline $^{19}$ or (dimethylamino)benzonitrile ${ }^{20}$ are also active for SHG. para-Nitroaniline-loaded MCM-41 also shows a SHG activity that is comparable to that of potassium dihydrogen phosphate (KDP) powders whose $d_{33}$ (a tensor component of the quadratic nonlinear susceptibility) is $\sim 3 \mathrm{pm} / \mathrm{V}^{24}$ For them to be SHG active, however, the water-assisted secondary reorganization of the included para-nitroaniline molecules is necessary to give rise to a net bulk dipole moment of the para-nitroaniline-loaded MCM-41. However, in the case of Sb-incorporating silicalite-1 loaded with para-nitroaniline, the SHG activity disappears after several exposures to incident laser beams. Unlike ZSM-5 and Sb-incorporating silicalite-1 which contain $\mathrm{Al}$ and $\mathrm{Sb}$, respectively, in the framework, the closely related pure silica ZSM-12 does not show any SHG activity even after inclusion of para-nitroaniline. ${ }^{23}$

As mentioned earlier, the primary demerit is that only para-nitroaniline and the analogous NLO dyes with relatively low $\beta$ values $\left(35 \times 10^{-30} \mathrm{esu}\right)^{14,15}$ spontaneously enter zeolite pores with uniform orientations. Furthermore, the zeolite hosts have been limited to powders that bear limited practical applicability.

As a means to develop a novel method to prepare practically useful 2O-NLO materials, we chose hemicyanine (HC) and its derivatives as the 2O-NLO dyes and have developed general methods for orientation-controlled inclusion of the $\mathrm{HC}$ dyes into zeolite channels. The $\beta$ value of $\mathrm{HC}\left(770 \times 10^{-30}\right.$ esu) is about 22 times higher than that of para-nitroaniline $\left(35 \times 10^{-30} \mathrm{esu}\right)$. For this purpose, a series of HC derivatives was prepared (Fig. 2). They are HC dyes with alkyl tails with various lengths (denoted as HC- $n$ with $n=3,6,9$, 12, 15, 18, 22, and 24), azo-bridged HC-9 (denoted as AZ-9), and the $\mathrm{HC}$ tethered with a propionic acid unit on the pyridinium side (denoted as $\mathrm{HC}-2-\mathrm{COOH}$ ). In the case of $\mathrm{HC}-n$ dyes the estimated maximum length of HC- $n$ dye increased from $1.7(n=3)$ to $4.2 \mathrm{~nm}(n=24)$ as $n$ increases (Table 1$).{ }^{25}$

As for the zeolite host, silicalite-1 films supported on glass plates were prepared (Fig. 3). In this film the straight channels (aperture size $=5.4 \times 5.6 \AA$ ) were oriented vertically (borientation) with respect to the glass supports. ${ }^{6}$ Although the kinetic diameter of the dyes is $\sim 5.79 \AA$, they can slowly enter the silicalite- 1 channels. In this Account we describe 


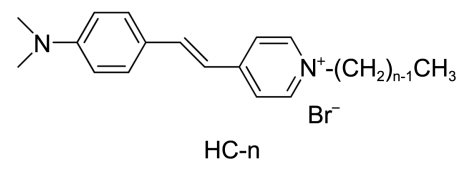

$\mathrm{n}=3,6,9,12,15,18,22$, and 24

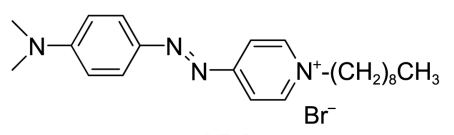

AZ-9

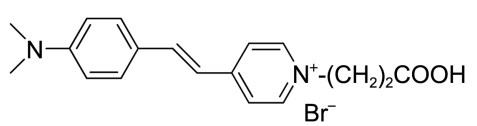

$\mathrm{HC}-2-\mathrm{COOH}$

Figure 2. Structures of HC derivatives that appear in this Account.

three strategies to control the orientation of the $\mathrm{HC}$ dyes during their inclusion into sililicate-1 films, namely, through the hydrophobic-hydrophobic interaction, inclusion of $\mathrm{HC}$ dyes during their excited states, and the size-excluded large cation as the orientation mediator.

Preparation of Silicalite-1 Films Grown on Both Sides of Glass Plates (SL/G/SL). The silicalite-1 films supported on glass plates were prepared by immersing glass plates $(25$ $\times 70 \times 1 \mathrm{~mm}^{3}$ ) into the gel consisting of tetraethylorthosilicate, tetrapropylammonium hydroxide, and water in the mole ratio of 0.8:0.1:50, followed by heating at $140{ }^{\circ} \mathrm{C}$ for $5 \mathrm{~h}$ in a Teflon-lined autoclave. The silicalite-1 films grew on both sides of glass plates with their b-axes (straight channels) orienting perpendicular to the glass planes (denoted as $\mathrm{SL} / \mathrm{G} /$ $\mathrm{SL}$ ). The SL/G/SL plates were washed with copious amounts of water and dried in the atmosphere at room temperature. The SL/G/SL plates were cut into four pieces with the size of $\sim 25 \times 18 \times 1 \mathrm{~mm}^{3}$, and calcined at $4500^{\circ} \mathrm{C}$ for $12 \mathrm{~h}$ to remove the encapsulated tetrapropylammonium ions prior to incorporation of NLO dyes. The film thickness of the silicalite-1 layer was $\sim 400 \mathrm{~nm}$.

Inclusion of HC-n dyes into SL/G/SL Plates. Into each vial $(25 \mathrm{~mL}$ capacity) containing a methanol solution of different $\mathrm{HC}-n(10 \mathrm{~mL}, 1 \mathrm{mM})$ two $\mathrm{SL} / \mathrm{G} / \mathrm{SL}$ plates were added, and the vial was kept at room temperature for a

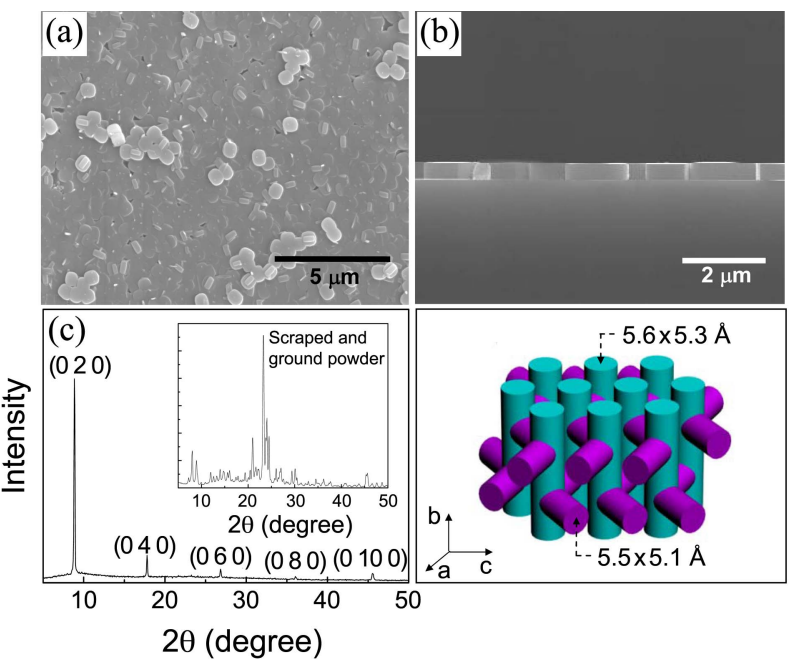

Figure 3. SEM images of a typical SL/G/SL plate, top (a) and cross sectional (b) views and X-ray diffraction pattern of the SL/G/SL plate (c). The diffraction lines appeared at a regular interval $(2 \theta=$ $8.85,17.8,26.85,34.85$, and $\left.45.55^{\circ}\right)$ corresponding to $(020),(04$ $0),\left(\begin{array}{lll}0 & 6 & 0\end{array}\right),\left(\begin{array}{lll}0 & 8 & 0\end{array}\right)$, and ( $\left(\begin{array}{lll}0 & 10 & 0\end{array}\right)$ planes of the crystals. Inset in C: The X-ray powder diffraction pattern of the scraped and ground film. This confirms that the film is indeed comprised of pure silicalite-1. (d) Illustration of channel structures in silicalite-1.

desired period of time from 1 day to 3 weeks. After equilibration, the SL/G/SL plates were removed from the solution, washed with copious amounts of fresh methanol, and dried in the air. The SL/G/SL plates incorporating HC- $n$ are denoted as $[\mathrm{HC}-n @ \mathrm{SL}]_{2} / \mathrm{G}$. The included number of each HC- $n$ molecule per channel $\left(N_{\mathrm{C}}\right)$ after dipping a SL/G/SL plate in $1 \mathrm{mM}$ solution of each HC- $n$ for 1 week (Table 1). $N_{C}$ increases upon increasing $n$ from 3 (6.4) to 6 (15.4) but rapidly decreases upon further increasing $n$ and finally reached to $\sim 1$ when $n=22$ and 24 .

The included dyes do not come out to the solution if they once enter the silicalite- 1 channels. For instance, the intensities of the visible bands of $[\mathrm{HC}-3 @ \mathrm{SL}]_{2} / \mathrm{G}$ and $[\mathrm{HC}-$ $18 @ \mathrm{SL}]_{2} / \mathrm{G}$ at $479 \mathrm{~nm}$ do not decrease even after keeping them in fresh methanol for 3 days, and the UV-vis spectra of the supernatant solutions also do not show any indication of

Table 1. Effect of alkyl chain length (n) on the optical properties of HC- $n$ and the amount of inclusion into silicalite- 1 film of $400-\mathrm{nm}$ thickness. Adopted from ref 25

\begin{tabular}{rccccccccccccc}
\hline$n$ & $\lambda_{\max }{ }^{a}$ & $\varepsilon^{b}$ & length $^{\mathrm{c}}$ & $N\left(\times 10^{14}\right)^{d}$ & $N_{C}{ }^{e}$ & $I_{p p}{ }^{f}$ & $I_{s p}\left(\times 10^{-4}\right)^{f}$ & $d_{33^{g}}{ }^{g} d_{31^{g}}$ & $d_{33} / d_{31}$ & $d_{33} / N_{\mathrm{C}}$ & $S^{h}$ & $\mathrm{DUO}^{i}$ \\
\hline 3 & 475.5 & 39515 & 1.7 & 4.64 & 6.4 & 0.3 & - & 1.12 & - & - & 0.18 & - & $0.08^{j}$ \\
6 & 479.0 & 43355 & 2.1 & 15.56 & 23.1 & 0.1 & - & 0.50 & - & - & 0.02 & - & $0.01^{j}$ \\
9 & 479.0 & 43775 & 2.4 & 10.37 & 15.4 & 1.6 & 0.5 & 2.25 & 0.02 & 113 & 0.15 & 0.97 & 0.09 \\
12 & 480.0 & 42460 & 2.8 & 5.70 & 8.2 & 3.8 & 2.2 & 3.59 & 0.04 & 90 & 0.63 & 0.97 & 0.20 \\
15 & 479.5 & 45160 & 3.1 & 4.30 & 5.7 & 7.0 & 1.7 & 4.99 & 0.04 & 125 & 0.88 & 0.98 & 0.38 \\
18 & 479.5 & 44340 & 3.5 & 2.59 & 3.5 & 7.9 & 3.0 & 5.30 & 0.05 & 106 & 1.51 & 0.98 & 0.66 \\
22 & 479.0 & 43180 & 4.0 & 0.61 & 0.9 & 0.9 & 0.4 & 1.71 & 0.02 & 86 & 1.90 & 0.97 & 0.91 \\
24 & 479.0 & 42320 & 4.2 & 0.88 & 1.2 & 1.9 & 0.9 & 2.57 & 0.03 & 86 & 2.14 & 0.97 & 0.95 \\
\hline
\end{tabular}

${ }^{a} \mathrm{In} \mathrm{nm} .{ }^{b}$ Molar extinction coefficient in MeOH, $\mathrm{M}^{-1} \mathrm{~cm}^{-1} .{ }^{c} \mathrm{In} \mathrm{nm} .{ }^{d}$ The total number of HC- $n$ in a SL/G. ${ }^{e}$ Number of HC- $n$ in each 400-nm long channel

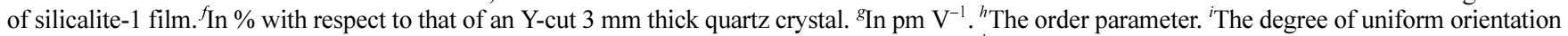
(DUO) of HC- $n$, defined by the experimental-to-theoretical ratio of $d_{33}$ values, $d_{33}(\mathrm{E}) / d_{33}(\mathrm{~T}) .{ }^{j}$ The average orientational angle $\left(\theta=7.7^{\circ}\right)$ was taken to derive the theoretical $d_{33}$ values. 
HC-3 and HC-18. The shows that the silicalite- 1 channel has a very strong affinity toward $\mathrm{HC}-n$ dyes regardless of the chain length, indicating that inclusion of HC- $n$ into the silicalite- 1 channel is non-equilibrium, irreversible process in methanol.

Inclusion of HC-9 into SL/G/SL Plates in the Photoexcited State. A rectangular, double-walled stainless steel chamber with the internal dimension of $20 \times 10 \times 35 \mathrm{~mm}^{3}$ was prepared. The chamber had four confronting quartz widows, a water inlet, and an outlet (Fig. 4(a)). A Teflon support $\left(20 \times 10 \times 3 \mathrm{~mm}^{3}\right)$ having a groove with the size of $10 \times 1 \times 1 \mathrm{~mm}^{3}$ was placed at the bottom of the container so that the direction of the groove become parallel with the direction of beam passage. A methanol solution of HC-9 (30 $\mathrm{mL}, 0.1 \mathrm{mM}$ ) was introduced into the chamber and the solution was cooled to $5{ }^{\circ} \mathrm{C}$ by passing cold water through the jacket. A line-polarized laser beam (cw, $488 \mathrm{~nm}$, beam size $=3 \mathrm{~mm}$ ) from an $\mathrm{Ar}^{+}$-ion laser was passed through the quartz windows. Since the molar extinction coefficient of $\mathrm{HC}-9$ at $488 \mathrm{~nm}$ is $42,570 \mathrm{M}^{-1} \mathrm{~cm}^{-1}$, irradiation of HC-9 molecules with the laser beam ensures photoexcitation of the molecules. A SL/G/SL plate $(10 \times 25 \times 1 \mathrm{~mm})$ was quickly inserted into the groove of the Teflon support. This allowed the formation of 3-mm wide beam envelopes on both silicalite-1 films along the SL/G plate (Fig. 4(b), (c)). The beam intensity was varied from 0.0 to $2.0 \mathrm{~W}$. The polarization was either parallel (p-polarization) or perpendicular (s-polarization) to the direction of the channels. After $6 \mathrm{~h}$, the SL/G/SL plate was quickly removed from the solution, washed with fresh methanol, and dried by blowing dry $\mathrm{N}_{2}$ over the plate. The number of molecules that enter each channel $\left(N_{\mathrm{C}}\right)$ in methanol at $5{ }^{\circ} \mathrm{C}$ remained constant $(\sim 13)$ regardless of the power of laser beam from 0.0 (dark) to 2.0 W.

Inclusion of HC-2-COOH into SL/G/SL Plates. Seven vials $(30 \mathrm{~mL}$ capacity) were filled with each $10 \mathrm{~mL}$ aliquot of the aqueous $\mathrm{HC}-2-\mathrm{COOH}$ dye solution $(0.1 \mathrm{mM})$. Aqueous TBAOH solutions (or $\mathrm{NaOH}$ or $\mathrm{NH}_{4} \mathrm{OH}$ ) with varying concentrations $(0.0,0.1,0.2,0.5,1.0,2.0,3.0 \mathrm{mM})$ were prepared and $10 \mathrm{~mL}$ of a TBAOH solution was added into the $\mathrm{HC}-2-\mathrm{COOH}$ dye containing vials so that [TBAOH] varied from 0 to $3.0 \mathrm{mM}$. SL/G/SL plates $(\sim 20 \times 18 \times 1$ $\mathrm{mm}^{3}$ ) were added into every vial, and vials were kept still at room temperature for 1 day. The $[\mathrm{HC}-2-\mathrm{COOH} @ \mathrm{SL}]_{2} / \mathrm{G}$ plates were removed from the vials, washed with copious amounts of fresh water, and dried in the air.

Measurement of SH Intensity of HC-incorporating SL/ G/SL Plates. The fundamental laser pulses (1064 nm, 40-ps pulse width, and $10-\mathrm{Hz}$ repetition rate) generated from a Continuum PY61 mode-locked Nd-YAG laser were used as the incident beam. A drop of DMSO was placed onto each side of a [HC@SL] $/ \mathrm{G}$, respectively, as an index matching fluid, and each side of the [HC@SL]2/G was covered with a clean bare glass plate $\left(25 \times 18 \times 1 \mathrm{~mm}^{3}\right)$. This is necessary to avoid scattering of the incident laser beam caused by the irregular thickness of the silicalite-1 films. A beam splitter and a photodiode were used to compensate for the intensity fluctuations of the 1064-nm beam. The polarity of the fundamental laser beam was adjusted using a half-wave plate before it hits the sample. The electric field vector of the incident beam was either parallel (p-polarization) or perpendicular (s-polarization) to the plane of incidence. Only the ppolarized SH beam was made to enter a PMT by using a prism and a SH pass filter. A $[\mathrm{HC} @ S L]_{2} / \mathrm{G}$ plate was mounted on the rotator coupled to a step motor. The output signals from the photodiode and PMT were detected as a function of an incident angle. A 3-mm-thick Y-cut quartz crystal (a piece of quartz plate whose plane is perpendicular to the crystalline $y$-axis) ${ }^{52}$ was used as a reference for determining the relative intensities of the $\mathrm{SH}$ signals generated from the samples. The SH signals were collected from three different spots of a [HC@SL $]_{2} \mathrm{G}$ plate, and the average intensity was taken. The relative $\mathrm{SH}$ intensities (expressed in \% with respect to an Y-cut 3-mm thick quartz) are more specifically expressed as $I_{p p}$ and $I_{s p}$, depending on the polarization direction of the incident beam, where the former and the latter denote the intensities of p-polarized SH beams generated from the p- and s-polarized fundamental laser beam, respectively.

Degree of Uniform Orientation (DUO). The experimentalto-theoretical ratio of $d_{33}$ (a tensor component of quadratic (a)

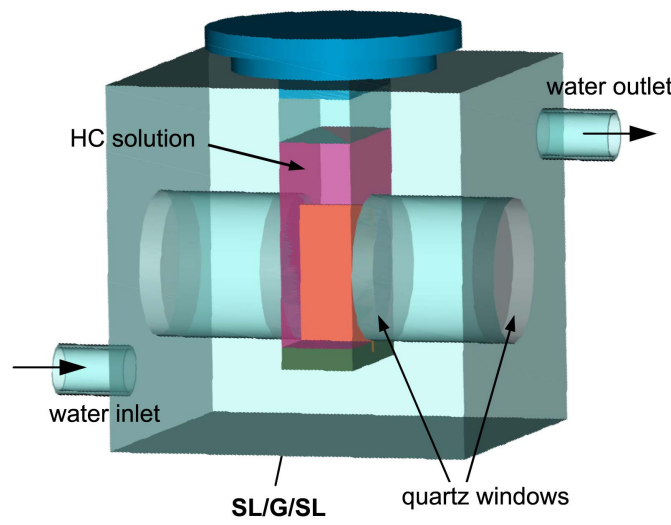

(b)

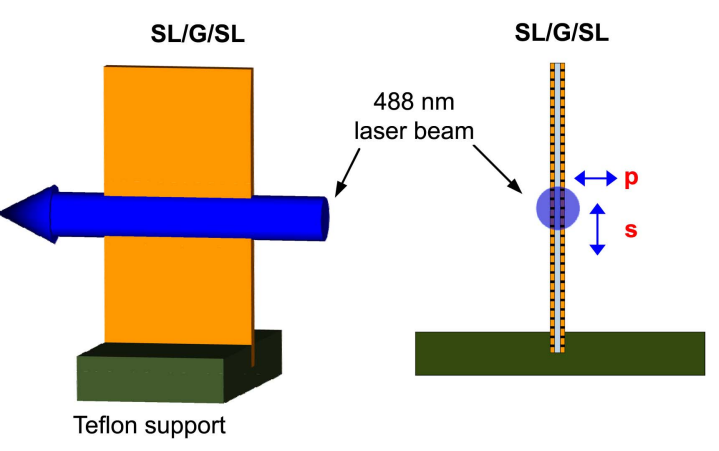

Figure 4. Illustration of a water-cooled irradiation chamber loaded with a SL/G plate and HC- $n$ solution (a) and the details of the irradiation of a SL/G plate with a 488-nm laser beam (beam diameter: $3 \mathrm{~mm}$ ) in two different views (b and c). Reproduced from ref 27. 

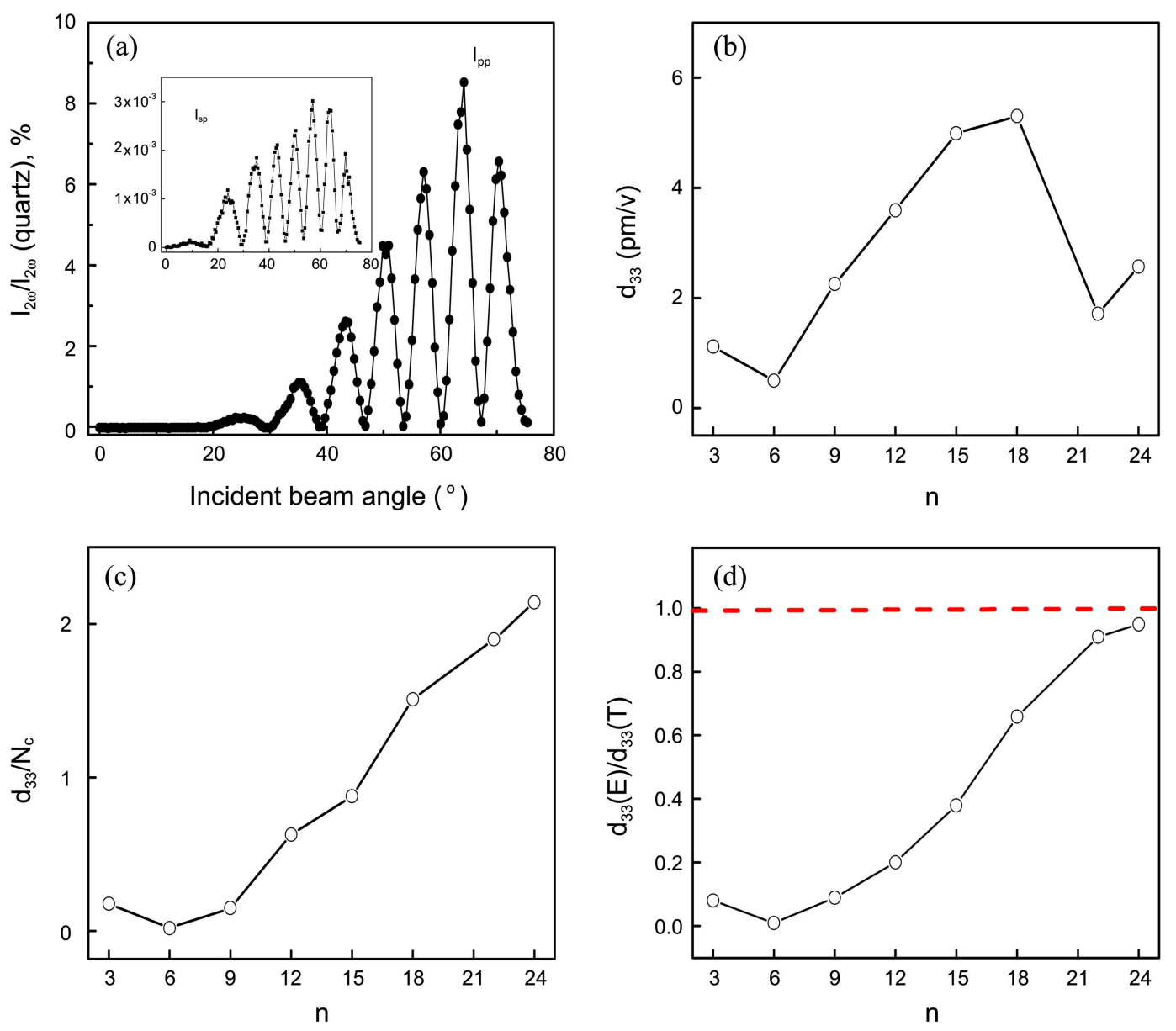

Figure 5. The plots of $I_{p p}$ and $I_{s p}$ (inset) of [HC-18@SL $]_{2} / \mathrm{G}$ (in \%, with respect to the corresponding value of Y-cut $3 \mathrm{~mm}$ quartz) versus the angle of the incident beam (a) and the plots of $d_{33}(\mathrm{~b}), d_{33} / N_{\mathrm{C}}(\mathrm{c})$, and $d_{33}(\mathrm{E}) / d_{33}(\mathrm{~T})$ (d) versus n. Reproduced from ref 25.

nonlinear susceptibility) $\left[d_{33}(\mathrm{E}) / d_{33}(\mathrm{~T})\right]$ is defined as the degree of uniform orientation (DUO). The DUO value is an important indicator to show the percentage of the NLO dyes that are incorporated into the silicalite-1 channels with uniform orientations increases.

Orientation Control by Hydrophobic-Hydrophobic Interaction between Alkyl Chain Tails in HC and Silicalite-1 Channels. $^{25}$

Inclusion into Silicalite-1 Channels with Hydrophobic Tail First: The typical plots of $I_{p p}$ and $I_{s p}$ of the [HC$18 @ \mathrm{SL}]_{2} / \mathrm{G}$ plate versus the angle of the incident beam (with respect to the surface normal) are shown in Figure 5(a). The complete destructive interferences in the profiles indicate that the two SL films on the opposite sides of glass plates are nearly identical. The maximum values of $I_{p p}$ and $I_{s p}$ appear at $67.4^{\circ}$ and $55.9^{\circ}$, respectively.

The $I_{p p}$ values ranged from 0.3 to $7.9 \%$ while those of $I_{s p}$ ranged from $0.4 \times 10^{-4}$ to $3.0 \times 10^{-4} \%$ (Table 1 ). Thus, $I_{p p}$ values are $\sim 10^{4}$ times larger than the $I_{s p}$ values, indicating that most of the dyes are positioned vertically with the long axis perpendicular to the glass plane. This is consistent with the fact that the straight channels are oriented vertically with respect to the glass plane (Fig. 6). The $I_{p p}$ value progressively increases from 0.1 to $7.9 \%$ with increasing $n$ from 6 to 18 , despite the progressive decrease in $N_{\mathrm{C}}$. Interestingly, consistent with the sharp decrease of $N_{\mathrm{C}}, I_{p p}$ sharply decreases from 7.9 to 0.9 and $1.9 \%$ upon further increasing $n$ from 18 to 22 and to 24 . The $I_{s p}$ value also shows a similar trend with that of $I_{p p}$ with increasing $n$.

The two tensor components of quadratic nonlinear susceptibility, $d_{33}$ and $d_{31}$ of [HC- $\left.n @ S L\right]_{2} / \mathrm{G}$ plates, are listed in Table 1, and graphically displayed in Figure 5(b). Like $I_{p p}$, the $d_{33}$ value initially decrease with increasing $n$ from 3 to 6 , but progressively increase with increasing $n$ from 6 to 18 , but sharply decrease upon further increasing $n$. However, $d_{33} / N_{\mathrm{C}}$ progressively increased with increasing $n$ (Table 1 and Fig. 4(c)). This phenomenon is likely to arise as a result of the increase in the tendency of HC- $n$ molecule to enter the hydrophobic silicalite-1 channels with the hydrophobic tail first as the tail length increases (Fig. 5).

The calculated theoretical maximum $d_{33}$ values $\left[d_{33}(T)\right]$ were 14.40 (3), 70.71 (6), 24.48 (9), 18.13 (12), 13.31 (15), 8.03 (18), 1.88 (22), and $2.71 \mathrm{pm} / \mathrm{V}(24)$, respectively, for each $n$ shown in the parenthesis. The DUO values are listed in Table 1 (last entry) and they are plotted against $n$ in Figure 5(d). Thus, DUO generally increases with increasing $n$. Such a phenomenon arises as a result of the increase in the tendency of HC- $n$ molecule to enter the hydrophobic silicalite-1 


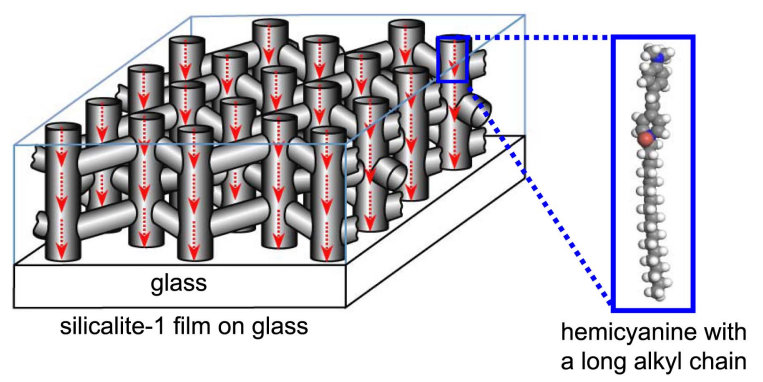

Figure 6. Schematic illustration of a silicalite-1 film incorporating HC- $n$ dyes with long alkyl chains within the hydrophobic tails uniformly pointing downward to the substrate caused by the incorporation of the dye into the silicaite- 1 channels with the long hydrophobic tails first. Adopted from ref 25.

channels with the hydrophobic tail first as $n$ increases. This phenomenon is also responsible for the progressive increase of $d_{33} / N_{\mathrm{C}}$ with increasing $n$ (Fig. 5(c)). In support of the above, the included amount of HC-3 into silicalite-1 powder was $0.01 \mathrm{mg}(0.3 \mu \mathrm{mol})$ per gram of silicalite- 1 while that of $n$-octadecane into silicalite-1 powders per gram of silicalite-1 was $3.64 \mathrm{mg}(143 \mu \mathrm{mol})$, after stirring the mixture for $3 \mathrm{~h}$ in each methanol solution. Thus, the included number of $n$ octadecane was as much as 476 times larger than that of HC3 within the period of $3 \mathrm{~h}$, despite the fact that the estimated length of $n$-octadecane (2.4 nm) is longer than that of HC-3 $(1.7 \mathrm{~nm})$.

The SH intensity of [HC-18@SL $]_{2} / \mathrm{G}$ increases parabolically as $N_{\mathrm{C}}$ of HC-18 increases. Thus, the observed $\mathrm{SH}$ intensities were $0.7,1.6,2.6,4.7$, and $7.5 \%$ for $N_{\mathrm{C}}$ of 1.2 , $1.7,2.2,3.0$, and $3.8^{53}$, respectively (Fig. 7). Knowing the fact that SHG intensity is proportional to the square of the number density of the NLO dye, the above result indicates that HC-18 molecules enter the silicalite- 1 channels with the same DUO regardless of the degree of loading.

The $d_{33}$ value of $[\mathrm{HC}-18 @ \mathrm{SL}]_{2} / \mathrm{G}\left(5.3 \mathrm{pmV}^{-1}\right)$ is more than 10 times larger than $d_{11}$ of quartz $\left(0.3 \mathrm{pmV}^{-1}\right)$, or the $d_{33}$ value of $\operatorname{KDP}\left(\sim 3 \mathrm{pmV}^{-1}\right)$, despite the fact that there are only 3.5 molecules in each channel. However, the observed $d_{33}$ values are much smaller than those observed from LB films, which are in the range of 35 and $750 \mathrm{pmV}^{-1}$. 1,2,4-8 The reason for the $d_{33}$ values of HC- $n$-SL/Gs being smaller than those of self-assembled molecular films is that $400 \mathrm{~nm}$ was taken as the thickness of the silicalite- 1 film. Since only 3.5 molecules are occupying the silicalite- 1 film and it can be said that $12.3 \mathrm{~nm}$ is the real thickness occupied by HC-18. In such a case the $d_{33}$ value becomes $172 \mathrm{pmV}^{-1}$. In the case of $[\mathrm{HC}-24 @ \mathrm{SL}]_{2} / \mathrm{G}, N_{\mathrm{C}}$ is essentially one. While $d_{33}$ value becomes $2.57 \mathrm{pmV}^{-1}$ when $400 \mathrm{~nm}$ is taken as the thickness of the film, it becomes $245 \mathrm{pmV}^{-1}$ when $4.2 \mathrm{~nm}$ is taken as the thickness of the film.

[HC-n@SL $]_{2} / \mathrm{G}$ plates have long-term thermal and mechanical stabilities, which most of the LB and poled polymer NLO films are lacking. ${ }^{1,2}$ For instance, HC- $n$-SL/Gs retained their initial SHG activities even after keeping them in the atmosphere for 1 year or in an oven at $120^{\circ} \mathrm{C}$ for $24 \mathrm{~h}$, and even after rubbing the surface with fingers many times,

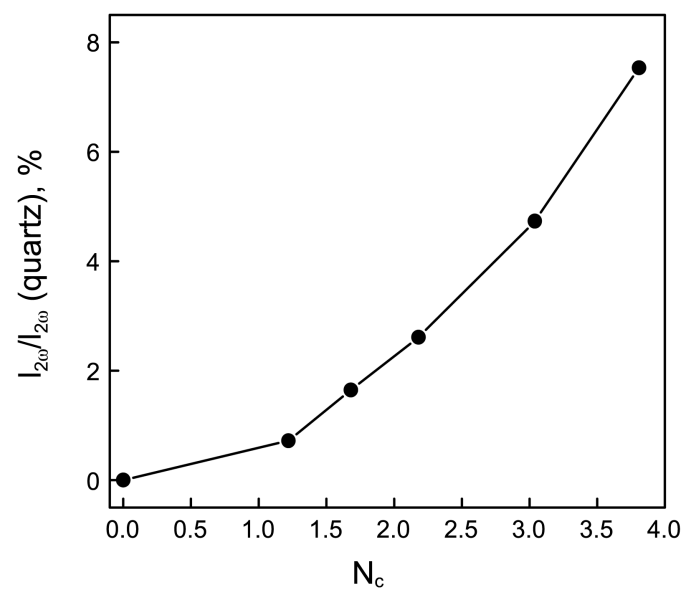

Figure 7. The parabolic relationship between the number of $\mathrm{HC}-8$ dye in each channel $\left(N_{\mathrm{C}}\right)$ and the SH intensity of [HC-18@SL] $/ \mathrm{G}$. Reproduced from ref 25 .

indicating the great potential of the NLO-dye-including zeolites to be developed into practically viable SHG materials.

Very High Order Parameters of HC- $n$ Dyes in SL/Gs: The average $d_{33} / d_{31}$ ratio was 109 which is $\sim 2-5$ times higher than those of Langmuir-Blodget (LB) films of nonlinear optical (NLO) dyes, and $\sim 30-50$ times higher than those $(\sim 3)$ of poled polymers imbedded or grafted with NLO dyes. ${ }^{1}$ The estimated average tilted angle of HC- $n(\theta)$ in the SL channel based on the above very high average $d_{33} / d_{31}$ ratio was $7.7^{\circ},{ }^{54}$ indicating that the long axes of the hemicyanine heads are tilted by the angle from the direction of the straight channels of silicalite-1 film (surface normal). The degree of orientational order of nonlinear chromophores can be quantified by the order parameter $\mathrm{s}$ which is expressed by Eq. (1).

$$
s=\left[3\left\langle\cos ^{2} \theta\right\rangle-1\right] / 2
$$

From the derived average $\theta$ of $7.7^{0},{ }^{54}$ the average order parameter (s) of HC- $n$ in SL/G/SL plates becomes 0.97, is markedly higher than those of NLO dyes in poled polymers $(\sim 0.2){ }^{1,2}$ The $s$ value of HC- $n$ in SL/G/SL plates is highest ever found among the SHG materials comprised of organic NLO dyes.

Inclusion of HC dyes with Medium Alkyl Chain Lengths into Silicalite-1 Channels in Photoexcited State. ${ }^{26} \mathrm{HC}-n$ dyes with $n \geq 18$ have a great tendency to enter the silicalite-1 channels with the alkyl chain first (Fig. 8(a)), giving rise to high DUOs $(>0.67)$. However, shorter-chain HCs such as HC-9 shows a poor DUO (0.08), because the hydrophilic center is localized at the pyridinium part and both the 1,4(dimethylamino)phenylenevinyl and $n$-nonyl groups are almost equally hydrophobic (Fig. 8(b)), thereby allowing the molecule to enter the channels with either the alkyl (Fig. 8(c)), or the dimethylamino part (Fig. 8(d)) first with almost no preference.

In the case of HC, theoretical studies have shown that, in the excited state, a substantial amount of positive charge density was generated at the dimethylamine terminal whilst 
(a)

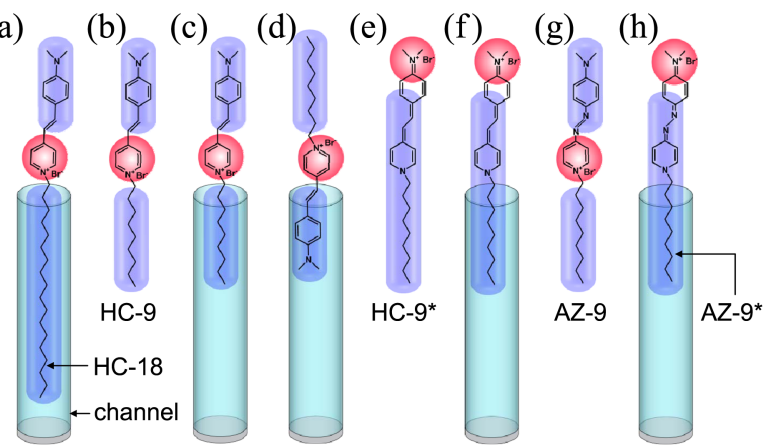

Figure 8. Schematic illustration of the initial step of HC-18 inclusion into a hydrophobic silicalite-1 channel with the hydrophobic alkyl chain first (a), presence of two hydrophobic parts on both sides and one hydrophilic part at the center in HC-9 (b), the inclusion of HC-9 into a channel with the alkyl (c) and dimethylamine (d) part first, localization of a hydrophilic part at the terminal in HC-9* (e), inclusion of HC-9* into a channel with the alkyl part first (f), presence of two hydrophobic parts on both sides and one hydrophilic part at the center in AZ-9 (g), and inclusion of AZ-9* into a channel with the alkyl part first (h). Reproduced from ref 26.

almost all of the positive charge density disappears from the pyridinium ring (Fig. 8(e))..$^{55}$ This leads to the shift of the hydrophilic part from the center to the very end of the molecule. Therefore, it is expected that a larger fraction of photoexcited HC-9 (HC-9*) molecules would enter the channels with the alkyl part first (Fig. 8(f)), as if it became a longer chain $\mathrm{HC}$.

The relative $I_{2 \omega}$ (at $70^{\circ}$ of the Maker fringe) with respect to that of 3-mm thick Y-cut quartz $\left[I_{2 \omega} / I_{2 \omega}(q z) \times 100\right.$, in \%] almost linearly increased from 1.5 to $10 \%$ with increasing the laser power from 0.0 to $1.6 \mathrm{~W}$ and then slightly decreased upon further increasing the power to $2.0 \mathrm{~W}$ (Fig. 9(a)). ${ }^{56}$ This means that DUO increased almost linearly from 0.08 to 0.27 (a 3.4-fold increase) with increasing the laser power from 0.0 to $1.6 \mathrm{~W}$ (Fig. 9(a)). Calculation shows that 1.25 molecules of 6 molecules which enter each channel with the dimethylamine side part first in the dark change orientation to the alkyl side upon irradiation at $1.6 \mathrm{~W}$, a $21 \%$ increase. Comparison with the DUOs of HC-12 (0.2) and HC-15 $(0.38)$ in the dark suggests that HC-9* ${ }^{*}$ behaves like HC-13.

The ratio of $I_{2 \omega}$ with light on with respect to that with light off $\left[I_{2 \omega}\right.$ (light on) $/ I_{2 \omega}$ (light off)] is 9.2 and 2.0 for p- and spolarization direction, respectively (Fig. 9(b)). This indicates that those molecules that are oriented parallel to the channel direction in the vicinity of the silicalite-1 film mostly enter the channels in the excited state (Fig. 8(f)). The measured $N_{\mathrm{C}}$ of the related AZ-9 (Fig. $8(\mathrm{~g})$ ) in methanol at $5{ }^{\circ} \mathrm{C}$ after $24 \mathrm{~h}$ (8.1, regardless of irradiation at $1.6 \mathrm{~W})$ was still smaller than that of HC-9 ( 13), indicating that the inclusion rate of AZ-9 was lower than that of HC-9. The photoexcited AZ-9 (AZ$9^{*}$ ) preferentially enters channels with a higher preference to the alkyl side (Fig. 8(h)).

This result not only opens new areas of research on the achievement of higher DUOs by photoexcitation and the study of its effect on the dynamics and kinetics of molecular diffusion at the solution-zeolite interfaces. In fact, the inclu-
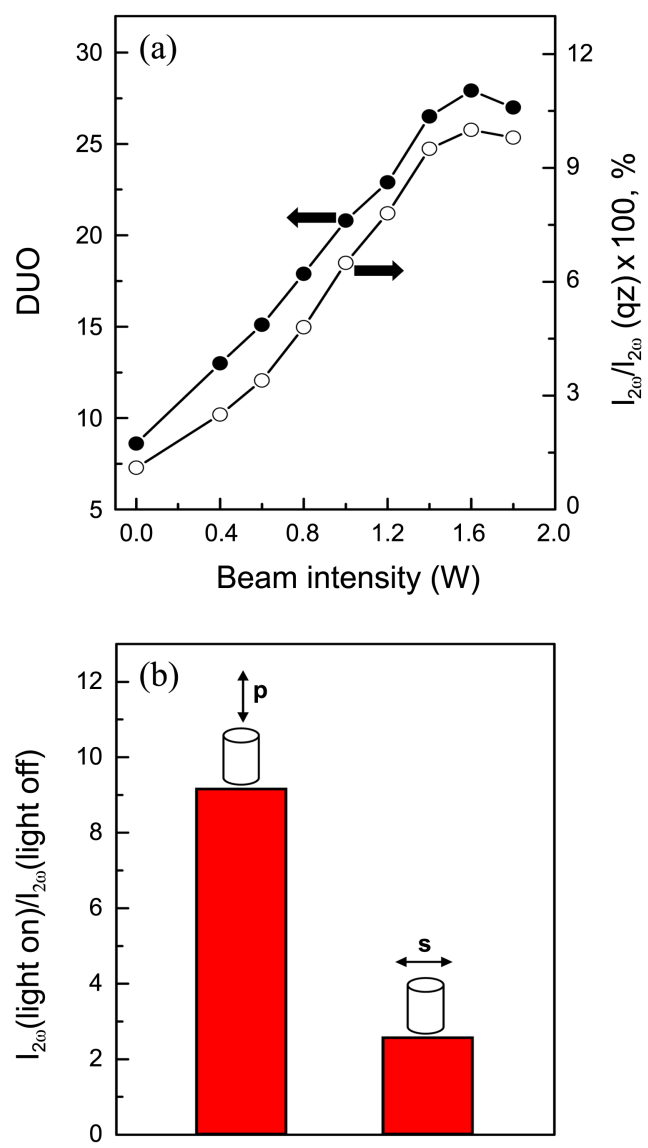

Figure 9. Plots of the DUO and $\left[I_{2 \omega} / I_{2 \omega}(q z) \times 100\right]$ (in \%) with respect to the laser beam intensity (a) and the effect of the beam polarization direction (p or s) on the $I_{2 \omega}$ (light on) to $I_{2 \omega}$ (light off) ratio (b). Reproduced from ref 27.

sion of molecules into zeolite channels in the photoexcited state has never been reported. ${ }^{26}$

Orientation Control by Ion Pairing of the $\boldsymbol{n}$-Propionic Acid-Tethering HC. ${ }^{27}$ In the case of HC- $n, N_{\mathrm{C}}$ increases upon decreasing $\mathrm{n}$. However, the decrease in $\mathrm{n}$ led to a sharp decrease in SH intensity due to the decrease in DUO. Thus, the method to increase both $N_{\mathrm{C}}$ and DUO has to be developed. An approach was to incorporate $\mathrm{HC}-2-\mathrm{COOH}$ into SL channels in the presence of tetrabutylammonium hydroxide (TBAOH), leading to a $\sim 16$-fold increase in $\mathrm{SH}$ intensity.

The SH intensities ( $I_{2 \omega}$ values) of [HC-2-COOH@SL $]_{2} / \mathrm{G}$ plates prepared from the solutions of $\mathrm{NaOH}$ and $\mathrm{NH}_{4} \mathrm{OH}$ remain constant at $\sim 1.0 \%$ (with respect to that of Y-cut 3mm thick quartz) regardless of the concentration of $\mathrm{NaOH}$ and $\mathrm{NH}_{4} \mathrm{OH}$ in the concentration range of 0.0 to $3.0 \mathrm{Mm}$ (Fig. 10(a)). Thus, the presence of $\mathrm{NaOH}$ or $\mathrm{NH}_{4} \mathrm{OH}$ into the aqueous solution of $\mathrm{HC}-2-\mathrm{COOH}$ does not cause the increase in DUO of HC-2-COOH in [HC-2-COOH@SL $]_{2} /$ G. In contrast, however, $I_{2 \omega}$ values of [HC-2-COOH@SL] $/$ G plates prepared from the solutions of $\mathrm{TBAOH}$ progressively increase from $1.0 \%$ to $16.0 \%$ as the concentration of TBAOH increases from 0 to $2.0 \mathrm{mM}$, and reached a plateau 

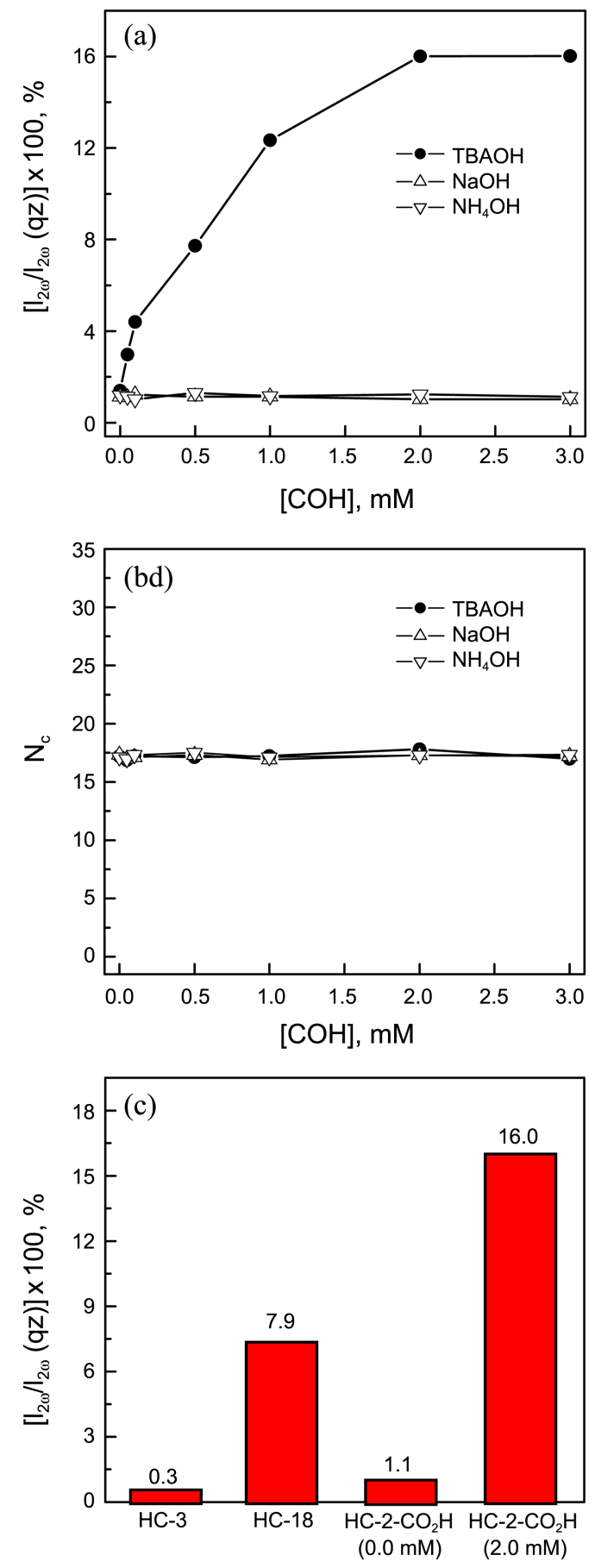

Figure 10. The plot of $I_{2 \omega}$ (a) and $N_{\mathrm{C}}$ (b) $v s$. [TBAOH], [NaOH], and $\left[\mathrm{NH}_{4} \mathrm{OH}\right]$, as indicated. Comparison of $I_{2 \omega} \mathrm{S}$ of $[\mathrm{HC}-3 @ \mathrm{SL}]_{2} / \mathrm{G}$, $[\mathrm{HC}-18 @ \mathrm{SL}]_{2} / \mathrm{G}$, and $[\mathrm{HC}-2-\mathrm{COOH} @ \mathrm{SL}]_{2} / \mathrm{G}$ at $[\mathrm{TBAOH}]$ of 0.0 and $2.0 \mathrm{mM}$, respectively (c). Adopted from ref 27 .

when the concentration $\geq 2.0 \mathrm{mM}$. Interestingly, the $N_{\mathrm{C}}$ values are invariably $\sim 16$ per channel, regardless of the concentrations of TBAOH, $\mathrm{NaOH}$, and $\mathrm{NH}_{4} \mathrm{OH}$ during the inclusion of HC-2-COOH into SL/G/SL plates (Fig. 10(b)). Since $I_{2 \omega}$ depends on $N_{\mathrm{C}}{ }^{2}$ aligned in a uniform orientation, the ratio of DUO of HC-2-COOH with the TBAOH concentration of $2.0 \mathrm{mM}$ to that of $\mathrm{HC}-2-\mathrm{COOH}$ with no TBAOH should be $(\sim 16 / 1)^{1 / 2}$, i.e., $\sim 4$. Thus, TBAOH (at $2.0 \mathrm{mM}$ ) (a)

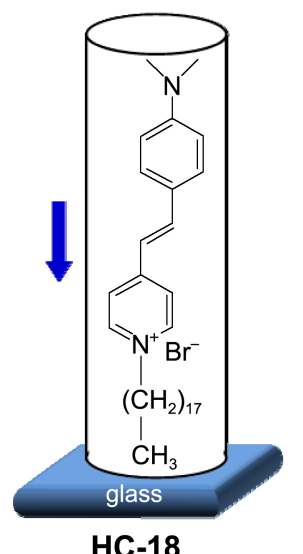

HC-18 (b)

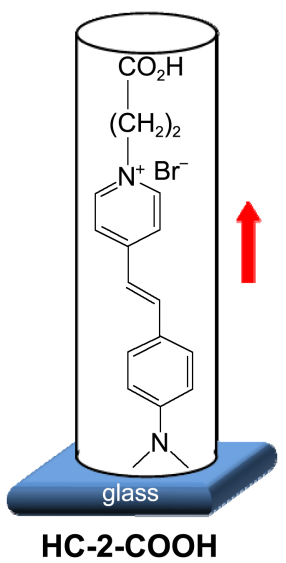

Figure 11. Structures of the orientations of HC-18 (a) and HC-2$\mathrm{COOH}(\mathrm{b})$ in SL/G plates. Adopted from ref 27.

induces a $\sim 4$-fold increase in DUO of the $\mathrm{HC}-2-\mathrm{COOH}$ in SL channels.

The $I_{2 \omega}$ values of $[\mathrm{HC}-2-\mathrm{COOH} @ \mathrm{SL}]_{2} / \mathrm{G}$ plates at the TBAOH concentration of 0.0 and $2.0 \mathrm{mM}$, respectively, are compared in Figure 10(c) with those of [HC-3@SL] $/$ /G and [HC-18@SL $]_{2} / \mathrm{G}$ plates and HC-18. Thus, $I_{2 \omega}$ of [HC-2$\mathrm{COOH} @ \mathrm{SL}]_{2} / \mathrm{G}$ obtained at the TBAOH concentration of $2.0 \mathrm{mM}$ is larger than those of $[\mathrm{HC}-18 @ \mathrm{SL}]_{2} / \mathrm{G}$ and $[\mathrm{HC}-$ $3 @ \mathrm{SL}_{2} / \mathrm{G}$ plates by 2 and 57 times, respectively. The $d_{33}$ value of $\left[\mathrm{HC}-2-\mathrm{COOH} @ \mathrm{SL}_{2} / \mathrm{G}\left(7.5 \mathrm{pmV}^{-1}\right)\right.$ is larger than the $d_{11}$ value of quartz by $\sim 25$ times than the $d_{33}$ value of KDP by $\sim 3$ times.

The 4-fold increase in DUO with increasing [TBAOH] from 0 to $2.0 \mathrm{mM}$ further shows that orientation control by ion pairing of $\mathrm{HC}-2-\mathrm{COOH}$ with bulky $\mathrm{TBA}^{+}$ion is less effective than that by hydrophobic-hydrophobic interaction between the long tail of $\mathrm{HC}$ and the SL channels, indicating that the higher $I_{2 \omega}$ of $[\mathrm{HC}-2-\mathrm{COOH} @ \mathrm{SL}]_{2} / \mathrm{G}$ plates at $[\mathrm{TBAOH}]=2.0 \mathrm{mM}$ arises from the much higher $N_{\mathrm{C}}$ of HC2-COOH in each SL channel (16) than that of HC-18 in each SL channel (3.5).

An independent phase measurement study ${ }^{57}$ revealed that a larger fraction of HC-2-COOH molecules enter the SL channels with dimethylaniline side first (Fig. 11). Thus the marked increase in DUO and the preferred 'head-down' orientation of HC-PA indicate that the following series of reactions (eqs. 2-4) occur.

$$
\begin{gathered}
\mathrm{HC}-2-\mathrm{COOH}+\mathrm{TBAOH}=\mathrm{HC}-2-\mathrm{COOTBA}+\mathrm{H}_{2} \mathrm{O} \\
\mathrm{HC}-2-\mathrm{COOTBA}+{ }_{\mathrm{g}}[]_{\mathrm{e}}={ }_{\mathrm{g}}\left[\mathrm{HC}-2-\mathrm{COO}^{-}\right]_{\mathrm{e}} \mathrm{TBA}^{+}
\end{gathered}
$$

${ }_{\mathrm{g}}\left[\mathrm{HC}-2-\mathrm{COO}^{-}\right]_{\mathrm{e}} \mathrm{TBA}^{+}+\mathrm{H}_{2} \mathrm{O}={ }_{\mathrm{g}}[\mathrm{HC}-2-\mathrm{COOH}]_{\mathrm{e}}+\mathrm{TBAOH}(4)$

Thus, HC-2-COOH and TBAOH undergo acid-base titration leading to the formation of HC-2-COOTBA and $\mathrm{H}_{2} \mathrm{O}$ (eq. 2). Due to $\mathrm{TBA}^{+}$(kinetic diameter of $8.1 \AA$ ), which is larger than the SL channel aperture $(5.6 \times 5.3 \AA)$, only the anion part ( $\left.\mathrm{HC}-2-\mathrm{COO}^{-}\right)$of $\mathrm{HC}-2-\mathrm{COOTBA}$ ion pairs insert into SL channels with the dimethylaniline side pointing to 


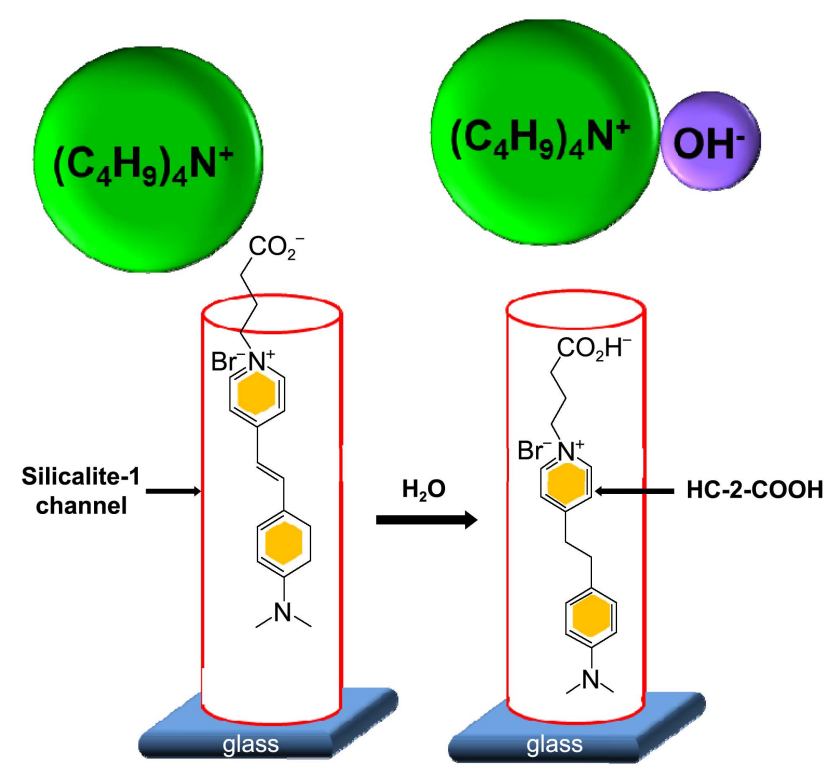

Figure 12. Schematic illustration of $\mathrm{HC}-2-\mathrm{COOH}$ molecules preferentially entering into the channels of silicalite-1 films with the head part first in water in the presence of TBAOH. Adopted from ref 27.

the glass substrate while keeping the cation $\left(\mathrm{TBA}^{+}\right)$outside the channel (eq. 3, where, ${ }_{g}[]_{e},{ }_{g}[\text {, and }]_{e}$ represent a channel, glass and entrance sides of the channel, respectively (Fig. 12). The encapsulated $\mathrm{HC}-2-\mathrm{COO}^{-}$then goes into the channel after acquiring a proton from water by undergoing the cation metathesis reaction (eq. 4).

Such a marked effect of $\mathrm{TBA}^{+}$is ascribed to the anisotropic size exclusion of HC (size exclusion of only one side of the molecule) by reversible salt formation of the tail part with $\mathrm{TBA}^{+}$. However, compared to the length of the SL channel $(\sim 400 \mathrm{~nm})$ the observed $N_{\mathrm{C}}(\sim 16)$ of HC-PA is still low (occupying only $\sim 8.5 \%$ of each channel space).

3O-NLO Zeolite Films by Inclusion of PbS QDs into Zeolite-Y Films. ${ }^{50}$

Preparation of Zeolite-Y Films on ITO Glass: Despite the fact that zeolites are excellent hosts for semiconductor $\mathrm{QDs}^{28-39}$ and QDs have shown 3O-NLO properties, the measurements of 3O-NLO properties of zeolite-encapsulated QDs could not be made due to the difficulty to prepare zeolite films supported on optically transparent substrates such as glass and quartz with reasonably high binding strengths between the films and substrates. For instance, when zeolite-Y films are grown on ordinary glass or fused silica, they readily peel off the substrates during drying, ionexchange with other ions, and calcination to remove organic contaminants. However, the zeolite-Y films grown on the surfaces of indium-tin-oxide-coated (ITO) glass plates remain firmly bonded to the substrates during ion exchange with $\mathrm{Pb}^{2+}$ ions, drying, and formation of $\mathrm{PbS}$ by treating $\mathrm{Pb}^{2+}$ ions with $\mathrm{H}_{2} \mathrm{~S}$ and that the encapsulated PbS QDs show very high 3O-NLO activities. ${ }^{50}$

Under the synthetic condition, the zeolite-Y films usually grow on ITO glass with the thickness of $2 \mu \mathrm{m}$. This is denoted as $\mathrm{Y} / \mathrm{G}$. The initially obtained $\mathrm{Y} / \mathrm{G}$ plates are opaque.

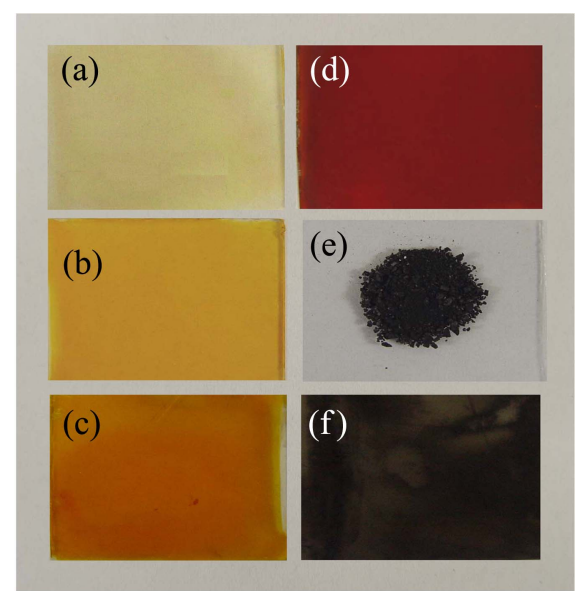

(g)

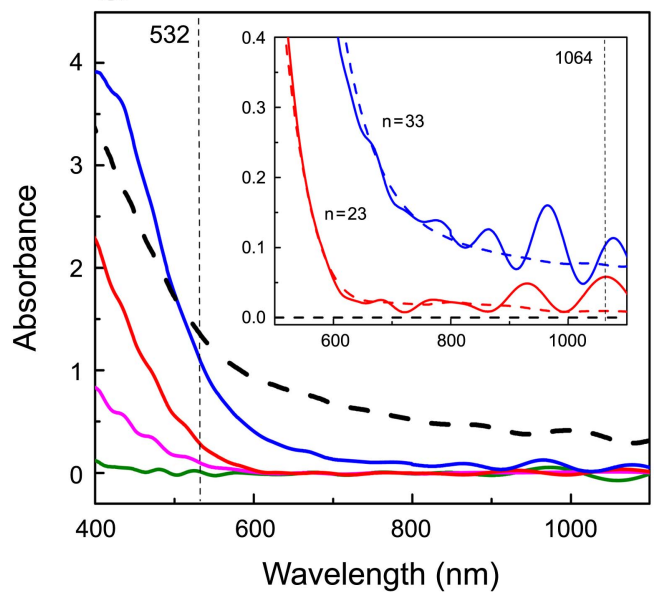

Figure 13. The photographs of ODM- $(\mathrm{PbS})_{\mathrm{n}}-\mathrm{Y} / \mathrm{G}$ for $n=8$ (a) 14 (b), 23 (c), and 33 (d), the bulk PbS powder on a $\mathrm{Y} / \mathrm{G}$ plate (e), moist $(\mathrm{PbS})_{33^{-}} \mathrm{Y} / \mathrm{G}(\mathrm{f})$, and absorption spectra $(\mathrm{g})$ of ODM- $(\mathrm{PbS})_{\mathrm{n}^{-}}$ $\mathrm{Y} / \mathrm{G}$ for $n=8$ (green), 14 (pink), 23 (red), and 33 (blue), and moist$(\mathrm{PbS})_{33}-\mathrm{Y} / \mathrm{G}$ (black dash). (Inset: Enlarged spectra of $n=23$ and 33 in the 500-1100 $\mathrm{nm}$ region. The dashed curves represent the corresponding estimated absorption spectra free from interference patterns). Adopted from ref 51.

Therefore, it is necessary to polish $\mathrm{Y} / \mathrm{G}$ plates until it becomes transparent. The thickness then reduces to $1.5 \mu \mathrm{m}$. Several polished $\mathrm{Y} / \mathrm{G}$ plates loaded with different numbers $(n=0,8$, 14,23 , and 33) of PbS in a unit cell were prepared [denoted as $\left.\left[(\mathrm{PbS})_{\mathrm{n}}\right] \mathrm{Y} / \mathrm{G}\right]$.

Tight Confinement of PbS QDs within Zeolite by Surface Silylation: The QDs are readily expelled from zeolite interior upon exposure of the $\left[(\mathrm{PbS})_{n}\right] \mathrm{Y} / \mathrm{G}$ to the ambient atmosphere due to the moisture adsorption. As a result, 3-20 nmsized PbS QDs are formed on the external surfaces accompanying zeolite framework destruction. This process leads to a significant loss of 3O-NLO activity of the $\left[(\mathrm{PbS})_{\mathrm{n}}\right] \mathrm{Y} / \mathrm{G}$. To prevent this phenomenon, the surfaces of dry $(\mathrm{PbS})_{n}-\mathrm{Y} / \mathrm{G}$ was coated with octadecyl groups using octadecyltrimethoxysilane (ODM) in a glove box charged with dry argon. ${ }^{48,50}$ The ODM-coating not only effectively prevents PbS QDs from leaving the internal pores but also leads to the formation of interconnected PbS QDs within zeolite-Y.4 ${ }^{48,50}$ The ODMcoated and moisture-exposed $\left[(\mathrm{PbS})_{\mathrm{n}}\right] \mathrm{Y} / \mathrm{G}$ are denoted as 
ODM- $\left[(\mathrm{PbS})_{\mathrm{n}}\right] \mathrm{Y} / \mathrm{G}$ and Moist- $\left[(\mathrm{PbS})_{\mathrm{n}}\right] \mathrm{Y} / \mathrm{G}$, respectively.

The color of ODM-[(PbS $\left.)_{n}\right] \mathrm{Y} / \mathrm{G}$ progressively red-shifts from pale yellow to deep red with increasing $n$ (Fig. 13(a)(d)). These colors are distinctively different from the color of bulk PbS, black (Fig. 13(e)). The color of moist-[(PbS) $]$ Y $/ \mathrm{G}$ plate is also black (Fig. 13(f)), due to the presence of large PbS QDs (3-20 nm) on the external surfaces. The absorption (Figure $13 \mathrm{G}$ ) spectra show that the amount of PbS QDs adsorbing $532 \mathrm{~nm}$ increases with increasing $n$. The enlarged spectra (inset) further reveal that the samples with $n=23$ and 33 contain intrazeolite QDs adsorbing even 1,064 nm, although the absorbance is very low ( $\mathrm{abs}=0.01$ and 0.07 , respectively). Moist- $\left[(\mathrm{PbS})_{\mathrm{n}}\right] \mathrm{Y} / \mathrm{G}$ shows absorption over the entire visible region and even beyond 1,100 nm (Fig. 13(g), black dashed curve), due to large PbS QDs existing on the external surfaces.

3O-NLO Responses: The measured nonlinear refraction $(\gamma)$ and absorption ( $\beta)$ coefficients of the films at 532 and $1,064 \mathrm{~nm}$, respectively, are shown in Table 2 . The active species are intrazeolite PbS QDs and their 3O-NLO activities occur by resonant processes (the responses that are triggered by absorption of $\mathrm{QD})$. The fact that moist- $\left[(\mathrm{PbS})_{33}\right] \mathrm{Y} / \mathrm{G}$ gave very weak $3 \mathrm{O}$ NLO response shows that the large external $\mathrm{PbS}$ QDs are not or weakly active in contrast to the intrazeolite PbS QDs.

The obtained $\gamma$ and $\beta$ values of ODM- $(\mathrm{PbS})_{33}-\mathrm{Y} / \mathrm{G}$ at 532 $\mathrm{nm}$ are larger by 47 and 118 times, respectively, in magnitude than the highest values ever observed from PbS QDs $\left(-6\right.$ and -50 , respectively) ${ }^{5}{ }^{5}$ and the values at $1064 \mathrm{~nm}$ are larger by 130 and 31 times, respectively, in magnitude than the highest values ever measured from PbS QDs ( -1 and 46, respectively) under similar input pulse conditions. ${ }^{58}$ The values at $1064 \mathrm{~nm}$ are still larger by 23 and 18 times than the values observed from GaAs dispersed in vycor glass ${ }^{59}$ which are the highest values ever observed from QDs.

The negative $\gamma$ values reveal that the PbS QDs show a selfdefocusing behavior at both wavelengths. The $\beta$ values are positive (at $532 \mathrm{~nm}$ ) and negative (at 1,064 nm) indicating that the PbS QDs behave as strong excited-state absorbers and optical bleachers, respectively, at the corresponding wavelengths. Opposite signs of $\beta$ were observed from larger $\mathrm{PbS}$ QDs dispersed in polyvinyl alcohol. ${ }^{58}$ The measured relaxation time of PbS QDs at $532 \mathrm{~nm}$ was less than $100 \mathrm{ps}$, which is short enough for them to be applied for optical

Table 2. $3 \mathrm{NLO}$ activities of $(\mathrm{PbS})_{\mathrm{n}}$-intercalating zeolite- $\mathrm{Y}$ films. Adopted from ref 51

\begin{tabular}{ccccc}
\hline ODM- $\left[(\mathrm{PbS})_{\mathrm{n}}\right] \mathrm{Y} / \mathrm{G}$ & \multicolumn{2}{c}{$532 \mathrm{~nm}$} & \multicolumn{2}{c}{$1064 \mathrm{~nm}$} \\
\cline { 2 - 5 }$n$ & $\gamma^{a}$ & $\beta^{b}$ & $\gamma^{a}$ & $\beta^{b}$ \\
\hline 8 & - & - & - & - \\
14 & -11 & 85 & - & - \\
23 & -34 & 290 & -32 & -72 \\
33 & $-294(6)^{c}$ & $5900(-50)^{c}$ & $-130(-1)^{c}$ & $1440(-46)^{c}$ \\
33 (moist) & - & 45 & - & - \\
\hline
\end{tabular}

$a_{\times} 10^{-12} \mathrm{~cm}^{2} / \mathrm{W} .{ }^{b} \mathrm{~cm} / \mathrm{GW} .{ }^{c}$ Highest literature values (ref 58 ). switching devices.

Figure of Merits: The figures of merit of ODM- $\left[(\mathrm{PbS})_{33}\right] \mathrm{Y} /$ $\mathrm{G}\left[\chi^{(3)} / \alpha_{0}\right.$ and $\gamma / \alpha_{0}$, where $\alpha_{0}=$ linear absorption coefficient $]$ are $3.4 \times 10^{-12}$ esu'cm and $-4 \times 10^{-14} \mathrm{~cm}^{2} / \mathrm{W} \cdot \mathrm{cm}$ at $532 \mathrm{~nm}$ and $2.3 \times 10^{-11}$ esu $\cdot \mathrm{cm}$ and $-2.8 \times 10^{-13} \mathrm{~cm}^{2} / \mathrm{W} \cdot \mathrm{cm}$ at 1,064 $\mathrm{nm}$, respectively. Comparison with the $\gamma / \alpha_{0}$ value reliably obtained from 2.3-3.5 nm PbS QDs dispersed in silicatitania film $\left(-5 \times 10^{-15} \mathrm{~cm}^{2} / \mathrm{W} \cdot \mathrm{cm}\right)^{60}$ which was measured under similar conditions ( $60 \mathrm{ps}$ pulse width at $1,064 \mathrm{~nm}$ ), the $\gamma / \alpha_{0}$ value of ODM- $\left[(\mathrm{PbS})_{33}\right] \mathrm{Y} / \mathrm{G}$ is at least 56 times higher.

The fact that the $3 \mathrm{O}-\mathrm{NLO}$ activities of ODM- $\left[(\mathrm{PbS})_{\mathrm{n}}\right] \mathrm{Y} / \mathrm{G}$ occur by resonant processes indicates that the activities originate from the laser-induced transient changes in the absorption spectra. ${ }^{28}$ The followings are proposed to cause the very high 3O-NLO activities. The sizes of QDs $(<1.5$ $\mathrm{nm})$ are smaller than those that have been dispersed in other media $(>2.5 \mathrm{~nm})$ due to encapsulation in zeolite pores. Second, zeolite pores can accommodate large number of QDs without transforming them to larger QDs due to the rigidity of the pore systems. Third, the strong electric field within the pores greatly stabilizes the generated excitons, which also provides the opportunity to form even biexcitons, whose formation is favored with decreasing the QD size. ${ }^{29}$

Summary and Future Perspectives. The orientations of $\mathrm{HC}$ dyes can be controlled during their incorporation into silicaite-1 films supported on glass plates. This leads to the formation of 2O-NLO zeolite films which have long-term thermal and mechanical stabilities, which most of the LB and poled polymer NLO films are lacking. ${ }^{28,29}$ Thus the HC-including silicalite-1 films have great potential to be developed into practically viable SHG materials. The three orientation control methods that have been tested are (1) hydrophobic-hydrophobic interaction between the long alkyl tails of HC and the silicalite-1 channels, (2) incorporation of $\mathrm{HC}$ dyes with the medium chain lengths in the photoexcited state, and (3) ion pairing of the carboxylic acid-tethering $\mathrm{HC}$. The critical problem is that the number of HC dyes in each silicalite-1 channel is too small. This seems to take place by the defect sites existing in the channels. Therefore, future study has to be focused on the development of the methods to increase the incorporated number of $\mathrm{HC}$ dyes in the channels.

The zeolite-encapsulated PbS QDs show very high 3ONLO activities. Since there are many different types of QDs and many different types of zeolites having different pore sizes, pore shapes, pore networking, framework compositions, and cations, the development of 3O-NLO materials with much higher sensitivities operable at various wavelengths is just a matter of time.

Thus, this Account describes a promising new direction to which the search for highly sensitive 2O-NLO and 3O-NLO materials has to be conducted and a new direction to which zeolite research and applications have to be expanded.

Acknowledgments. This work was supported by the Korea Center for Artificial Photosynthesis (KCAP) located in Sogang University funded by MEST through the National 
Research Foundation of Korea (NRFK) (NRF-2009C1AAA001-2009-0093879) and the acceleration program of the NRFK. H.S.K. also thanks the Sogang University Research Grant of 200910017.

\section{References}

1. Nalwa, H. S., Myata, S., Eds.; Nonlinear Optics of Organic Molecules and Polymers; CRC: Florida, 1997.

2. Gunter, P., Ed.; Nonlinear Optical Effects and Materials; Springer: Heidelberg, 2000.

3. (a) Eaton, D. F.; Anderson, A. G.; Tam, W.; Wang, Y. J. Am. Chem. Soc. 1987, 109, 1886-1888. (b) Tom, W.; Eaton, D. F.; Calabrese, J. C.; Williams, I. D.; Wang, Y.; Anderson, A. G. Chem. Mater. 1989, 1, 128-140. (c) Wang, Y.; Eaton, D. F. Chem. Phys. Lett. 1985, 120, 441-444. (d) Tomaru, S.; Zembutsu, S.; Kawachi, M.; Kobayashi, M. Chem. Commun. 1984, 1207-1208. (e) Weissbuch, I.; Lahav, M.; Leiserowitz, L.; Meredith, G. R.; Vanherzeeles, H. Chem. Mater. 1989, 1, 114-118.

4. Ashwell, G. J.; Hargreaves, R. C.; Baldwin, C. E.; Bahra, G. S.; Brown, C. R. Nature 1992, 357, 393-395.

5. Katz, H. E.; Scheller, G.; Putvinski, T. M.; Schilling, M. L.; Wilson, W. L.; Chidsey, C. E. D. Science 1991, 254, 1485-1487.

6. Kanis, D. R.; Ratner, M. R.; Marks, T. J. Chem. Rev. 1994, 94 , 195-242.

7. (a) Marks, T. J.; Pagani, G. A.; Facchetti, A.; Abbotto, A.; Beverina, L.; Boom, M. E.; Dutta, P.; Evmenenko, G. Chem. Mater. 2003, 15, 1064-1072. (b) Wang, G.; Zhu, P.; Marks, T. J.; Ketterson, J. B. Appl. Phys. Lett. 2002, 16, 2169-2171. (c) Milko, E.; Boom, M. E.; Evmenenko, G.; Marks, T. J. Adv. Funct. Mater. 2001, 11, 393397. (d) Zhao, Y. G.; Wu, A.; Lu, H. L.; Chang, S.; Lu, W. K.; Ho, S. T. Appl. Phys. Lett. 2001, 79, 587-589. (e) Facchetti, A.; Abbotto, A.; Beverina, L.; Boom, M. E.; Dutta, P.; Evmenenko, G.; Marks, T. J.; Pagani, G. A. Chem. Mater. 2002, 14, 4996-5005. (f) Wostyn, K.; Binnemans, K.; Clays, K.; Persoons, A. J. Phys. Chem. 2001, 105, 5169-5173. (g) Bénard, S.; Yu, P.; Audière, J. P.; Rivière, E.; Clément, R.; Guilhem, J.; Tchertanov, L.; Nakatani, K. J. Am. Chem. Soc. 2000, 122, 9444-9454. (h) Schwartz, H.; Mazor, M.; Khodorkovsky, V.; Shapiro, L.; Klung, J. T.; Kovalev, E.; Meshulam, G.; Berkovic, G.; Kotler, Z.; Efrima, S. J. Phys. Chem. $B$ 2001, 105, 5914-5921. (i) Lin, S.; Meech, S. R. Langmuir 2000, $16,2893-2898$.

8. (a) Yang, X.; Mcbranch, D.; Swanson, B.; Li, D. Angew. Chem. Int. Ed. 1996, 35, 538-560. (b) Huang, W.; Helvenston, M. Langmuir 1999, 15, 6510-6514

9. Dalton, L. R.; Harper, A. W.; Ghosn, R.; Steier, W. H.; Ziari, M.; Fetterman, H.; Shi, Y.; Mustacich, R. V.; Jen, A. K.-Y.; Shea, K. J. Chem. Mater. 1995, 7, 1060-1081.

10. Marder, S. R.; Klppelen, B.; Alex, K. Y.; Peyghambarian, N. Nature 1997, 388, 845-851.

11. Boom, M. E. Angew. Chem. Int. Ed. 1996, 41, 3363-3366.

12. Samyn, C.; Verbiest, T.; Persoons, A. Macromol. Rapid Commun. 2000, 21, 1-15

13. (a) Saadeh, H.; Yu, D.; Wang, M.; Yu, L. P. J. Mater Chem. 1999, 9, 1865-1873. (b) Steire, W. H. Chem. Phys. 1999, 245, 487-506. (c) Jiang, H.; Kakkar, A. K. J. Am. Chem. Soc. 1999, 121, 36573665. (d) Dalton, L. R. J. Mater. Chem. 1999, 9, 1905-1920.

14. Persoons, A.; Clays, K. Phys. Rev. Lett. 1991, 66, 2980-2983.

15. Stähelin, M.; Burland, D. M.; Rice, J. E. Chem. Phys. Lett. 1992, 191, 245-250.

16. Cox, S. D.; Gier, T. E.; Stucky, G. D.; Bierlein, J. J. Am. Chem. Soc. 1988, 110, 2986-2987.

17. (a) Cox, S. D.; Gier, T. E.; Stucky, G. D. Chem. Mater. 1990, 2, 609-619. (b) Cox, S. D.; Gier, T. E.; Stucky, G. D.; Bierlein, J. Solid State Ionics 1989, 32, 514-520.

18. Marlow, F.; Wübbenhorst, M.; Caro, J. Phys. Chem. 1994, 98, 12315-12319.
19. Caro, J.; Marlow, F.; Hoffmann, K.; Kornatowski, J.; Girnus, I.; Noack, M.; Kölsch, P. Progress in Zeolite Microporous Materials 1997, 105, 2171-2178.

20. Marlow, F.; Caro, J.; Werner, L.; Kornatowski, J.; Dähne, S. J. Phys Chem. 1993, 97, 11286-11290.

21. Werner, L.; Caro, J.; Finger, G.; Kornatowski, J. Zeolite 1992, 12, 658-663.

22. Reck, G.; Marlow, F.; Kornatowski, J.; Hill, W.; Caro, J. J. Phys. Chem. 1996, 100, 1698-1704.

23. Kinski, I.; Daniels, P.; Deroche, C.; Marler, B.; Gies, H. Microporous Mesoporous Materials 2002, 56, 11-25.

24. Kinski, I.; Gies, H.; Marlow, F. Zeolites 1997, 19, 375-381.

25. Kim, H. S.; Lee, S. M.; Ha, K.; Jung, C.; Lee, Y.-J.; Chun, Y. S.; Kim, D.; Rhee, B. K.; Yoon, K. B. J. Am. Chem. Soc. 2004, 126, 673-682.

26. Kim, H. S.; Pham, T. T.; Yoon, K. B. J. Am. Chem. Soc. 2008, 130 , 2134-2135.

27. Kim, H. S.; Sohn, K. W.; Jeon, Y.; Min, H.; Kim, D.; Yoon, K. B. Adv. Mater. 2007, 19, 260-263.

28. Wang, Y. Acc. Chem. Res. 1991, 24, 133-139.

29. Hu, Y. Z.; Lindberg, M.; Koch, S. W. Phys. Rev. B 1990, 42, 17131723.

30. Guerreiro, P. T.; Ten, S.; Borrelli, N. F.; Butty, J.; Jabbour, G. E.; Peyghambarian, N. Appl. Phys. Lett. 1997, 71, 1595-1597.

31. Wang, Y.; Wang, M.; Yao, X.; Kong, F.; Zhang, L. J. Cryst. Growth 2004, 268, 575-579.

32. (a) Justus, B. L.; Tonucci, R. J.; Berry, A. D. Appl. Phys. Lett. 1992, 61, 3151-3153. (b) Dvorak, M. D.; Justus, B. L.; Berry, A. D. Opt. Lett. 1995, 116, 149-152.

33. Zhu, Y.; Elim, H. I.; Foo, Y.-L.; Yu, T.; Liu, Y.; Ji, W.; Lee, J.-Y.; Shen, Z.; Wee, A. T.-S.; Thong, J. T.-L.; Sow, C.-H. Adv. Mater. 2005, 18, 587-592.

34. Zhu, Y.; Elim, H. I.; Foo, Y.-L.; Yu, T.; Liu, Y.; Ji, W.; Lee, J.-Y.; Shen, Z.; Wee, A. T.-S.; Thong, J. T.-L.; Sow, C.-H. Adv. Mater. 2005, 18, 587-592.

35. Yu, B.; Yin, G.; Zhu, C.; Gan, F. Opt. Mater. 1998, 11, 17-21.

36. Lu, S. W.; Sohling, U.; Mennig, M.; Schmidt, H. Nanotechnology 2002, 13, 669-673.

37. Liu, B.; Li, H. P.; Chew, C. H.; Que, W. X.; Lam, Y. L.; Kam, C. H.; Gan, L. M.; Xu, G. Q. Mater. Lett. 2001, 51, 461-469.

38. Huang, W.; Shi, J. J. Mater. Res. 2000, 15, 2343-2346.

39. Martucci, A.; Fick, J.; Schell, J.; Battaglin, G.; Guglielmi, M. $J$ Appl. Phys. 1999, 86, 79-87.

40. Wang, Y.; Herron, N. J. Phys. Chem. 1987, 91, 257-260.

41. Moller, K.; Eddy, M. M.; Stucky, G. D.; Herron, N.; Bein, T. J. Am. Chem. Soc. 1989, 111, 2564-2571.

42. Stucky, G. D.; Mac Dougall, J. E. Science 1990, 247, 669-678

43. Liu, X.; Thomas, J. K. Langmuir 1989, 5, 58-66.

44. (a) Leiggener, C.; Calzaferri, G. Chem. Eur. J. 2005, 11, 71917198. (b) Leiggener, C.; Calzaferri, G. ChemPhysChem 2004, 5, 1593-1596.

45. Terasaki, O.; Yamazaki, K.; Thomas, J. M.; Ohsuna, T.; Watanabe, D.; Sanders, J. V.; Barry, J. C. Nature 1987, 330, 58-60.

46. Armand, P.; Saboungi, M.-L.; Price, D.-L.; Iton, L.; Cramer, C.; Grimsditch, M. Phys. Rev. Lett. 1997, 79, 2061-2064.

47. Ozin, G. A.; Steele, M. R.; Holmes, A. J. Chem. Mater. 1994, 6, 999-1010.

48. Jeong, N. C.; Kim, H. S.; Yoon, K. B. Langmuir 2005, 21, 6038.

49. Jeong, N. C.; Kim, H. S.; Yoon, K. B. J. Phys. Chem. C 2007, 111, 10298-10312.

50. Kim, H. S.; Jeong, N. C.; Yoon, K. B. J. Am. Chem. Soc. 2011, 133, 1642-1645

51. Kim, H. S.; Lee, M. H.; Jeong, N. C.; Lee, S. M.; Rhee, B. K.; Yoon, K. B. J. Am. Chem. Soc. 2006, 128, 15070-15071.

52. Quartz crystals generate $\mathrm{SH}$ along the y-axis. Therefore, a y-cut quartz is often used as a transmission SHG reference for Maker's fringe experiment. See, (a) Boyd, R. W. Nonlinear Optics, 2nd ed.; Academic: London, 2003; p 48. (b) Shen, Y. R. The Principles 
of Nonlinear Optics; Wiley: New York, 1988; 101.

53. The fact that this Nc value is higher than that shown in Table 1 is because SL/Gs were produced from a different batch.

54. To obtain the average tilted angle of HC- $n$ dyes in the silicalite- 1 channels, we assumed that the long axes of the hemicyanine heads are tilted with an average specific angle $\theta$ from the channel direction after inclusion into the straight channels running perpendicular to the glass plane. Then, $d_{31}$ and $d_{33}$ can be expressed in terms of $\beta_{\mathrm{HC}-n}$ as eqs. (1) and (2), respectively, by assuming that $\beta_{\mathrm{HC}-n}$ has only one dominant component $\beta=\beta_{\xi \xi \xi}$ along the longaxis direction $\xi$ (the direction of linear dipole moment), and the microscopic local field correction 1 is independent of direction and frequency (sample is isotropic and dispersionless),

$$
\begin{aligned}
& d_{33}=\left.\mathrm{N} \beta_{\mathrm{HC}-n}\left\langle\cos ^{3} \theta\right\rangle\right|^{3} \\
& d_{31}=\mathrm{N} \beta_{\mathrm{HC}-n}\left[\langle\cos \mathrm{q}\rangle-\left\langle\cos ^{3} \theta\right\rangle\right] 1^{3} / 2
\end{aligned}
$$

where, $\mathrm{N}$ is the number density of HC- $n$ (total number of HC- $n$ within a unit volume, which is $1 \mathrm{~cm} \times 1 \mathrm{~cm} \times 400 \mathrm{~nm}$ in our case, see Table 1$)$ and 1 is given by $\left(n^{2}+2\right) / 3$, where $\mathrm{n}$ is the refractive index of zeolite which was estimated to be 1.48 (see Text). The angle bracket indicates the orientational average of the molecules.
Since HC- $n$ dyes should have a very narrow distribution $\mathrm{f}(\theta)$ of orientation, like $f(\theta)=\delta \delta\left(\theta-\theta_{0}\right)$, as a result of confinement within the narrow straight channels of silicailte-1, we have the following relationship (eq. 3).

$$
d_{33} / d_{31}=2 / \tan ^{2} \theta=109
$$

From the above eq, $\mathrm{q}$ is derived to be 7.7.

55. (a) Huang, Y.; Cheng, T.; Li, F.; Huang, C.-H.; Hou, T.; Yu, A.; Zhao, X.; Xu, X. J. Phys Chem. B 2002, 106, 10020-10030. (b) Cao, X.; Tolbert, R. W.; Mchale, J. L.; Edwards, W. D. J. Phys Chem. A 1998, 102, 2739-2748.

56. The decrease of $I_{2 \omega}$ (and hence $\left.d_{33}\right)$ at a higher laser power $(2.0 \mathrm{~W})$ is ascribed to a large increase in local temperature.

57. Min, H.; Jeon, Y.; Sung, J. H.; Seok, S.; Kim, D.; Kim, H. S.; Yoon, K. B. J. Phys Chem. C 2007, 111, 18159-18163.

58. Yu, B.; Yin, G.; Zhu, C.; Gan, F. Opt. Mater. 1998, 11, 17-21.

59. (a) Justus, B. L.; Tonucci, R. J.; Berry, A. D. Appl. Phys. Lett. 1992, 61, 3151-3153. (b) Dvorak, M. D.; Justus, B. L.; Berry, A D. Opt. Lett. 1995, 116, 149-152.

60. Martucci, A.; Fick, J.; Schell, J.; Battaglin, G.; Guglielmi, M. J. Appl. Phys. 1999, 86, 79-87. 